\title{
Lie Algebras of Derivations and Resolvent Algebras
}

\author{
Detlev Buchholz ${ }^{a *}$ and Hendrik Grundling ${ }^{b}$ \\ ${ }^{a}$ Institut für Theoretische Physik and Courant Centre \\ "Higher Order Structures in Mathematics", Universität Göttingen, \\ 37077 Göttingen, Germany \\ ${ }^{b}$ Department of Mathematics, University of New South Wales \\ Sydney, NSW 2052, Australia
}

Dedicated to the memory of Hans--Jürgen Borchers

\begin{abstract}
This paper analyzes the action $\delta$ of a Lie algebra $X$ by derivations on a $\mathrm{C}^{*}$-algebra $\mathcal{A}$. This action satisfies an "almost inner" property which ensures affiliation of the generators of the derivations $\delta$ with $\mathcal{A}$, and is expressed in terms of corresponding pseudo-resolvents. In particular, for an abelian Lie algebra $X$ acting on a primitive $\mathrm{C}^{*}$-algebra $\mathcal{A}$, it is shown that there is a central extension of $X$ which determines algebraic relations of the underlying pseudoresolvents. If the Lie action $\delta$ is ergodic, i.e. the only elements of $\mathcal{A}$ on which all the derivations in $\delta_{X}$ vanish are multiples of the identity, then this extension is given by a (non-degenerate) symplectic form $\sigma$ on $X$. Moreover, the algebra generated by the pseudo-resolvents coincides with the resolvent algebra based on the symplectic space $(X, \sigma)$. Thus the resolvent algebra of the canonical commutation relations, which was recently introduced in physically motivated analyses of quantum systems, appears also naturally in the representation theory of Lie algebras of derivations acting on $\mathrm{C}^{*}$-algebras.
\end{abstract}

\section{Introduction and Framework}

In quantum physics, symmetry transformations are often given in terms of their infinitesimal action on the algebra of observables, i.e. as a Lie action of derivations on the observables. It is not always clear that there is a faithful representation of the observables in which the Lie algebra is represented by (selfadjoint) operators implementing the given Lie action by commutators. In fact, such covariant representations may not exist and one needs to take a cocycle representation of the Lie algebra to obtain the implementing property, or equivalently replace the Lie algebra by a central extension of it. Two prominent examples are first, the abelian group of position

${ }^{*}$ Supported by the German Research Foundation (Deutsche Forschungsgemeinschaft (DFG)) through the Institutional Strategy of the University of Göttingen 
and velocity transformations in quantum mechanics and second, the conformal transformations in two-dimensional quantum field theory. For these, the central extensions are the Heisenberg algebra and the Virasoro algebra, respectively. Which central extensions appear seems to be fixed by the structure of the underlying algebra $\mathcal{A}$. This fact has been observed in the context of quantum anomalies in many examples; but there does not yet exist a systematic investigation of it. It is the aim of the present article to begin such a study in a setting based on the following assumptions.

(I) Let $X$ be a (finite or infinite dimensional) real Lie algebra with Lie-bracket $[\cdot, \cdot]$ and let $\mathcal{A}$ be a unital $\mathrm{C}^{*}$-algebra which is primitive (i.e. it has a faithful irreducible representation). Let $\mathcal{A}_{0} \subset \mathcal{A}$ be a norm dense unital ${ }^{*}$-subalgebra, and let $\delta: X \rightarrow \operatorname{Der} \mathcal{A}_{0}$ be an injective Lie homomorphism into the Lie algebra $\operatorname{Der} \mathcal{A}_{0}$ of ${ }^{*}$-derivations of $\mathcal{A}_{0}$, i.e. $\delta$ is real linear and

$$
\delta_{f} \circ \delta_{g}-\delta_{g} \circ \delta_{f}=\delta_{[f, g]}, \quad f, g \in X .
$$

Such a pair $(X, \mathcal{A})$ will be called a Lie $C^{*}$-system. The action $\delta$ is said to be ergodic if $\delta_{f}\left(A_{0}\right)=0$ for all $f \in X$ implies that $A_{0}$ is a multiple of the identity.

This framework covers quantum physics, where algebras of observables are generally constructed in some distinguished irreducible representations, e.g. the Fock representation. On the other hand, it excludes classical physics, where the observable algebras are abelian and symmetries act in a non-trivial manner. The intermediate cases, where the underlying algebras of observables have a center on which $\delta$ acts trivially, such as in the presence of superselected charges, can usually be reduced to the present setting by proceeding to suitable quotient algebras.

Of particular interest for physics are Lie $\mathrm{C}^{*}$-systems where the action $\delta$ is induced by selfadjoint generators which can be interpreted as observables. The simplest case is if the derivations $\delta_{f}$ are inner, i.e. if for each $f \in X$ there are operators $G_{f}=G_{f}^{*} \in \mathcal{A}$ such that $\delta_{f}\left(A_{0}\right)=i\left[G_{f}, A_{0}\right]$ for all $A_{0} \in \mathcal{A}_{0}$. However, generically the generators of symmetries are unbounded operators and hence are not elements of the underlying $\mathrm{C}^{*}$-algebra. In order to see how to deal with these cases we rewrite the preceding equation in terms of the resolvents of the generators $G_{f}$ :

$$
\left(i \lambda \mathbf{1}+G_{f}\right)^{-1} \delta_{f}\left(A_{0}\right)\left(i \lambda \mathbf{1}+G_{f}\right)^{-1}=i\left[A_{0},\left(i \lambda \mathbf{1}+G_{f}\right)^{-1}\right]
$$

for $\lambda \in \mathbb{R} \backslash\{0\}$. This equation can be generalized so as to cover the case of unbounded generators which are affiliated with $\mathcal{A}$ by making use of the notion of pseudo-resolvent [7]; it replaces the familiar concept of the resolvent of a selfadjoint operator in the abstract $\mathrm{C}^{*}$-setting.

Definition: Let $\mathcal{A}$ be a $\mathrm{C}^{*}$-algebra. A pseudo-resolvent is a function $R: \mathbb{R} \backslash\{0\} \rightarrow \mathcal{A}$ such that

$$
R(\lambda)-R(\mu)=i(\mu-\lambda) R(\lambda) R(\mu), \quad R(\lambda)^{*}=R(-\lambda) \quad \text { for } \quad \lambda, \mu \in \mathbb{R} \backslash\{0\} .
$$

Any pseudo-resolvent can be analytically continued to the domain $\mathbb{C} \backslash i \mathbb{R}$. By some abuse of terminology, we will use the term pseudo-resolvent also for its values $R(z), z \in \mathbb{C} \backslash i \mathbb{R}$.

With this concept we can express the assumption that there exist (possibly unbounded) generators of the action $\delta$ which are affiliated with the algebra $\mathcal{A}$. 
(II) Let $(X, \mathcal{A})$ be a Lie $\mathrm{C}^{*}$-system. The underlying action $\delta$ is said to be almost inner if, for each $f \in X$, there is a pseudo-resolvent $R(\lambda, f) \in \mathcal{A}, \lambda \in \mathbb{R} \backslash\{0\}$ such that

$$
R(\lambda, f) \delta_{f}\left(A_{0}\right) R(\lambda, f)=i\left[A_{0}, R(\lambda, f)\right], \quad A_{0} \in \mathcal{A}_{0} .
$$

Remarks: It can be seen that relation (1.2) holds for all values of $\lambda$ if it holds for one. As a matter of fact, by analytic continuation in $\lambda$, it holds on the entire domain $\mathbb{C} \backslash i \mathbb{R}$. Moreover, the relation implies that $R(\lambda, 0)$ is contained in the center of $\mathcal{A}$ and hence must be a multiple of $\mathbf{1}$ since $\mathcal{A}$ is primitive. Assuming that this multiple is different from 0 , it follows from the defining relations of pseudo-resolvents that one can consistently put $R(\lambda, 0)=-(i / \lambda) \mathbf{1}, \lambda \in \mathbb{R} \backslash\{0\}$.

We will show below that condition (II) implies that $\delta$ is induced by selfadjoint generators in every faithful irreducible representation of $\mathcal{A}$. This observation is the key to analyzing the algebraic properties of the pseudo-resolvents inherited from the Lie structure of the derivations. For this analysis we need the following technical assumption which holds only for a restricted class of Lie algebras $X$, such as compact or abelian ones.

(III) Let $(X, \mathcal{A})$ be a Lie $\mathrm{C}^{*}$-system satisfying (II). For each $f \in X$, there is a pseudo-resolvent satisfying relation (1.2) which is in the domain $\mathcal{A}_{0}$, i.e. $R(z, f) \in \mathcal{A}_{0}$ for all $z \in \mathbb{C} \backslash i \mathbb{R}$.

Having stated the general framework, we now restrict the subsequent analysis to the simple but physically important case of abelian Lie algebras $X$. In this case the action $\delta$ is flat, i.e. the right hand side of equation (1.1) vanishes, and this explains the terminology used in the following definition.

Definition: Let $X$ be a real abelian Lie algebra. The pair $(X, \mathcal{A})$ is said to be a flat Lie $C^{*}{ }_{-}$system if it satisfies the conditions (I), (II) and (III).

Given any flat Lie $\mathrm{C}^{*}$-system $(X, \mathcal{A})$, we will determine the structure of the algebra $\mathcal{R} \subset \mathcal{A}$ generated by the associated pseudo-resolvents. It contains information about the commutation relations of the generators which implement the action $\delta$ and hence about the possible appearance of central extensions of $X$. We will see that for any such system there is a unique skew symmetric bilinear form $\sigma: X \times X \rightarrow \mathbb{R}$ fixing an extension. If $\delta$ acts ergodically on $\mathcal{A}$, the form $\sigma$ is nondegenerate and $(X, \sigma)$ is a symplectic space. The algebra $\mathcal{R}$ then coincides with the resolvent algebra $\mathcal{R}(X, \sigma)$, defined in [2]. Moreover, if $X$ is finite-dimensional, then its dimension must be even and the algebra $\mathcal{R}$ is the unique Heisenberg algebra of canonical commutation relations in resolvent form.

The article is organized as follows. The basic framework and assumptions are specified in this introduction. In Sect. 2 we work out the algebraic consequences, and establish existence of a skew symmetric bilinear form $\sigma$ on $X \times X$ entering into the commutation relations of the pseudoresolvents. In Sect. 3 we show by standard cohomological arguments that the pseudo-resolvents can be chosen in such a way that they also encode linearity of the action $\delta$ on $X$. We obtain therefore all the defining relations of the resolvent algebra on $(X, \sigma)$ and hence this algebra is a subalgebra of $\mathcal{A}$. We also show uniqueness of this subalgebra relative to the initial action. 


\section{Algebraic structure}

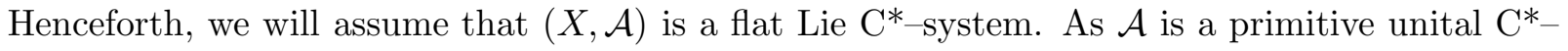
algebra, it has by definition a faithful irreducible representation. Thus we may assume without loss of generality that we have concretely $\mathcal{A} \subseteq \mathcal{B}(\mathcal{H})$ for some Hilbert space $\mathcal{H}$ and $\mathcal{A}^{-}=\mathcal{B}(\mathcal{H})$, where the bar denotes weak closure.

2.1 Lemma Let $f \in X$ and $\lambda \in \mathbb{R} \backslash\{0\}$.

(i) There is a selfadjoint operator (generator) $G_{f}$ with domain $\mathcal{D}_{f}=R(\lambda, f) \mathcal{H}$ such that $R(\lambda, f)=\left(i \lambda \mathbf{1}+G_{f}\right)^{-1}$.

(ii) For each $A_{0} \in \mathcal{A}_{0}$ there are $B_{0}, C_{0} \in \mathcal{A}_{0}$ such that

$$
A_{0} R(\lambda, f)=R(\lambda, f) B_{0} \quad \text { and } R(\lambda, f) A_{0}=C_{0} R(\lambda, f)
$$

Proof: (i) From relation (1.2) we see that $\operatorname{Ker} R(\lambda, f) \subset \mathcal{H}$, the kernel of $R(\lambda, f)$, is stable under the action of $\mathcal{A}_{0}$ and, by continuity, also under the action of $\mathcal{A}$. As $\mathcal{A}$ is irreducible, the kernel can only consist of $\{0\}$. However $\operatorname{Ker} R(\lambda, f)=\{0\}$ iff $R(\lambda, f)$ is a resolvent $R(\lambda, f)=\left(i \lambda+G_{f}\right)^{-1}$ by [5, Corollary 1], and $G_{f}$ has domain $\mathcal{D}_{f}=R(\lambda, f) \mathcal{H}$. Now $G_{f}$ is symmetric by

$$
G_{f}^{*}=\left(R(1, f)^{-1}-i \mathbf{1}\right)^{*} \supseteq i \mathbf{1}+\left(R(1, f)^{-1}\right)^{*}=i \mathbf{1}+R(-1, f)^{-1}=G_{f} .
$$

That it is also selfadjoint follows from the equality of ranges $\operatorname{Ran}\left( \pm i \mathbf{1}+G_{f}\right)^{-1}=\operatorname{Ran} R( \pm 1, f)$, the latter being dense by [5, Theorem on p. 467] since $\operatorname{Ker} R(\lambda, f)=\{0\}$.

(ii) It folows from relation (1.2) that

$$
A_{0} R(\lambda, f)=\left[A_{0}, R(\lambda, f)\right]+R(\lambda, f) A_{0}=R(\lambda, f)\left(-i \delta_{f}\left(A_{0}\right) R(\lambda, f)+A_{0}\right)
$$

But $B_{0} \doteq\left(-i \delta_{f}\left(A_{0}\right) R(\lambda, f)+A_{0}\right) \in \mathcal{A}_{0}$ since, by assumption, $\mathcal{A}_{0}$ is stable under the action of the derivations and the resolvents are elements of this algebra by condition (III). This proves the first part of the statement; the second part follows by a similar argument.

Note that $\mathcal{D}_{f}=R(\lambda, f) \mathcal{H}$ does not depend on $\lambda$. It is also noteworthy that the first part of the lemma holds for arbitrary Lie $\mathrm{C}^{*}$-systems satisfying (II), only in the second part did we use assumption (III). From this lemma we obtain:

2.2 Lemma Let $f \in X$. Then $\mathcal{A}_{0} \mathcal{D}_{f} \subset \mathcal{D}_{f}$ and

$$
\left[G_{f}, A_{0}\right] \Psi=-i \delta_{f}\left(A_{0}\right) \Psi, \quad A_{0} \in \mathcal{A}_{0}, \Psi \in \mathcal{D}_{f}
$$

Proof: It follows from part (ii) of the preceding lemma that $\mathcal{A}_{0} R(\lambda, f) \mathcal{H} \subset R(\lambda, f) \mathcal{H}$, proving the stability of the domain $\mathcal{D}_{f}$ under the action of $\mathcal{A}_{0}$. Now let $A_{0} \in \mathcal{A}_{0}$ and $\Psi \in \mathcal{D}_{f}$, i.e. 
$\Psi=R(\lambda, f) \Phi$ for some $\Phi \in \mathcal{H}$. Then, using computations in the preceding lemma,

$$
\begin{aligned}
& G_{f} A_{0} \Psi+i \lambda A_{0} \Psi \\
& =\left(i \lambda 1+G_{f}\right) A_{0} R(\lambda, f) \Phi \\
& =\left(i \lambda 1+G_{f}\right) R(\lambda, f)\left(-i \delta_{f}\left(A_{0}\right) R(\lambda, f)+A_{0}\right) \Phi \\
& =\left(-i \delta_{f}\left(A_{0}\right) R(\lambda, f)+A_{0}\right) \Phi \\
& =\left(-i \delta_{f}\left(A_{0}\right)+A_{0}\left(i \lambda 1+G_{f}\right)\right) R(\lambda, f) \Phi \\
& =\left(-i \delta_{f}\left(A_{0}\right)+A_{0} G_{f}\right) \Psi+i \lambda A_{0} \Psi,
\end{aligned}
$$

proving relation (2.1).

To analyze the commutation relations between the generators $G_{f}, G_{g}$, one needs more detail about their domains. The following result provides the relevant information.

2.3 Lemma Let $f, g \in X$ and let $\lambda, \mu \in \mathbb{R} \backslash\{0\}$. Then $\mathcal{D}_{f, g, f} \doteq R(\lambda, f) R(\mu, g) R(\lambda, f) \mathcal{H}$ is dense in $\mathcal{H}$. Moreover, $\mathcal{A}_{0} \mathcal{D}_{f, g, f} \subset \mathcal{D}_{f, g, f}$ and $\mathcal{D}_{f, g, f}$ is contained in the domains of $G_{f}, G_{g}$ as well as of their products in either order.

Proof: Since the resolvents are bounded and have dense range it follows that $\mathcal{D}_{f, g, f}$ is dense. Next, by threefold application of Lemma 2.1, there exists for any $A_{0} \in \mathcal{A}_{0}$ some $B_{0} \in \mathcal{A}_{0}$ such that $A_{0} R(\lambda, f) R(\mu, g) R(\lambda, f)=R(\lambda, f) R(\mu, g) R(\lambda, f) B_{0}$, proving the stability of $\mathcal{D}_{f, g, f}$ under the action of $\mathcal{A}_{0}$. Finally, it follows from its very definition that $\mathcal{D}_{f, g, f}$ lies in the domains of $G_{f}$ and $G_{g} G_{f}$; for the proof of the remaining assertion one makes use again of Lemma 2.1 which implies, bearing in mind that $A_{0} \doteq R(\lambda, f) \in \mathcal{A}_{0}$ according to condition (III),

$$
R(\lambda, f) R(\mu, g) R(\lambda, f)=R(\mu, g) B_{0} R(\lambda, f)=R(\mu, g) R(\lambda, f) C_{0}
$$

for certain specific operators $B_{0}, C_{0} \in \mathcal{A}_{0}$. Hence $\mathcal{D}_{f, g, f} \subset R(\mu, g) R(\lambda, f) \mathcal{H}$ also lies in the domains of $G_{g}$ and $G_{f} G_{g}$, completing the proof of the statement.

Below in Lemma 3.1 we will have to establish a stronger version of this lemma. Making use of the above result one can now compute the commutator of $\left[G_{f}, G_{g}\right]$ with the elements of $\mathcal{A}_{0}$.

2.4 Lemma Let $\Phi \in \mathcal{D}_{f, g, f}$ and let $A_{0} \in \mathcal{A}_{0}$. Then

$$
\left[G_{f}, G_{g}\right] A_{0} \Phi=A_{0}\left[G_{f}, G_{g}\right] \Phi
$$

Proof: The following computation relies on the preceding lemmata:

$$
\begin{aligned}
& G_{f} G_{g} A_{0} \Phi=G_{f}\left(-i \delta_{g}\left(A_{0}\right)+A_{0} G_{g}\right) \Phi \\
& =\left(-\delta_{f} \circ \delta_{g}\left(A_{0}\right)-i \delta_{g}\left(A_{0}\right) G_{f}-i \delta_{f}\left(A_{0}\right) G_{g}+A_{0} G_{f} G_{g}\right) \Phi .
\end{aligned}
$$

Interchanging $f$ and $g$ one obtains an analogous equality. By subtraction one arrives at

$$
\left[G_{f}, G_{g}\right] A_{0} \Phi=-\left(\delta_{f} \circ \delta_{g}-\delta_{g} \circ \delta_{f}\right)\left(A_{0}\right) \Phi+A_{0}\left[G_{f}, G_{g}\right] \Phi
$$

But $\delta_{f} \circ \delta_{g}-\delta_{g} \circ \delta_{f}=0$, completing the proof. 
It follows from this result and the assumption that $\mathcal{A}$ is irreducible that the commutator of $G_{f}, G_{g}$ is a c-number. The argument is based on a generalization of Schur's lemma adapted to unbounded operators. As it will be applied at various places, we recall here the well-known proof.

2.5 Lemma Let $f, g \in X$. There is a constant $\sigma(f, g) \in \mathbb{R}$, antisymmetric in $f, g$, such that

$$
\left[G_{f}, G_{g}\right] \Phi=i \sigma(f, g) \Phi \quad \text { for all } \Phi \in \mathcal{D}_{f, g, f}
$$

Proof: Let $K \doteq-i\left[G_{f}, G_{g}\right]$ on the domain $\mathcal{D}_{f, g, f}$. According to the preceding lemma we have $\left\langle\Psi, A_{0} K \Phi\right\rangle=\left\langle\Psi, K A_{0} \Phi\right\rangle$ for all $A_{0} \in \mathcal{A}_{0}$ and $\Phi, \Psi \in \mathcal{D}_{f, g, f}$. Taking also into account Lemma 2.3 and the fact that the generators are selfadjoint, we can proceed to $\left\langle\Psi, K A_{0} \Phi\right\rangle=\left\langle K \Psi, A_{0} \Phi\right\rangle$. As $\mathcal{A}_{0}$ is dense in $\mathcal{A}$ this implies that

$$
\langle K \Psi, A \Phi\rangle=\langle\Psi, A K \Phi\rangle, \quad A \in \mathcal{A}, \Phi, \Psi \in \mathcal{D}_{f, g, f}
$$

Now as $\mathcal{A} \subset \mathcal{B}(\mathcal{H})$ is irreducible, hence algebraically irreducible [6], for any given one-dimensional projection $E \in \mathcal{B}(\mathcal{H})$ and finite dimensional subspace $\mathcal{K} \subset \mathcal{H}$ there exists an operator $A_{E, \mathcal{K}} \in \mathcal{A}$ which acts like $E$ on $\mathcal{K}$, i.e. $A_{E, \mathcal{K}} \uparrow \mathcal{K}=E \uparrow \mathcal{K}$, cf. [4, Theorem 2.8.3(i)]. So one can replace in the above equality the operator $A$ by the projections onto the rays of $\Psi$ and $\Phi$, respectively (assuming $\Psi, \Phi \neq 0$ ), giving

$$
\langle\Psi, K \Phi\rangle=\langle\Psi, \Phi\rangle\langle\Psi, K \Psi\rangle /\|\Psi\|^{2}=\langle\Psi, \Phi\rangle\langle\Phi, K \Phi\rangle /\|\Phi\|^{2}
$$

As $K$ is a symmetric operator, it follows from this equality that $\sigma(f, g) \doteq\langle\Phi, K \Phi\rangle /\|\Phi\|^{2}$ is real and does not depend on the choice of $\Phi \in \mathcal{D}_{f, g, f}$. Hence $\langle\Psi, K \Phi\rangle=\sigma(f, g)\langle\Psi, \Phi\rangle$ and, since $\mathcal{D}_{f, g, f}$ is dense, this implies that $K \Phi=\sigma(f, g) \Phi$ for $\Phi \in \mathcal{D}_{f, g, f}$, as claimed. The antisymmetry of $\sigma(f, g)$ in $f, g$ follows from its definition.

This result allows us to establish corresponding algebraic properties of the resolvents.

2.6 Lemma Let $f, g \in X$. Then

$$
[R(\lambda, f), R(\mu, g)]=i \sigma(f, g) R(\lambda, f) R(\mu, g)^{2} R(\lambda, f), \quad \lambda, \mu \in \mathbb{R} \backslash\{0\} .
$$

Furthermore,

$$
\delta_{f}(R(\mu, g))=\sigma(f, g) R(\mu, g)^{2}, \quad \mu \in \mathbb{R} \backslash\{0\} .
$$

Proof: Let $\Phi \in \mathcal{D}_{f, g, f}$. Then, by condition (III) and Lemma 2.3, $R(\mu, g) R(\lambda, f) \Phi \in \mathcal{D}_{f, g, f}$ and one can compute

$$
\begin{aligned}
& i \sigma(f, g) R(\lambda, f) R(\mu, g)^{2} R(\lambda, f) \Phi \\
& =R(\lambda, f) R(\mu, g)\left[G_{f}, G_{g}\right] R(\mu, g) R(\lambda, f) \Phi \\
& =R(\lambda, f) R(\mu, g)\left[\left(i \lambda 1+G_{f}\right),\left(i \mu 1+G_{g}\right)\right] R(\mu, g) R(\lambda, f) \Phi \\
& =[R(\lambda, f), R(\mu, g)] \Phi .
\end{aligned}
$$


Since $\mathcal{D}_{f, g, f}$ is dense in $\mathcal{H}$ the first part of the statement follows. For the proof of the second part one makes use of condition (III), the preceding result and Lemma 2.2, giving

$$
\begin{aligned}
& -i \delta_{f}(R(\mu, g)) R(\lambda, f) \Phi \\
& =\left(i \lambda 1+G_{f}\right) R(\mu, g) R(\lambda, f) \Phi-R(\mu, g) \Phi \\
& =\left(i \lambda 1+G_{f}\right) R(\lambda, f)\left(R(\mu, g)-i \sigma(f, g) R(\mu, g)^{2} R(\lambda, f)\right) \Phi-R(\mu, g) \Phi \\
& =-i \sigma(f, g) R(\mu, g)^{2} R(\lambda, f) \Phi .
\end{aligned}
$$

Since the resolvent $R(\lambda, f)$ is bounded and has dense range, the space $R(\lambda, f) \mathcal{D}_{f, g, f}$ is dense in $\mathcal{H}$, and so the second part of the statement follows.

Remark: It is an immediate consequence of the second statement that the resolvents are analytic elements for the derivations.

The preceding lemma allows one to establish further properties of the form $\sigma$. Recall that $X$ is a real vector space, and by condition (I), the map $\delta: X \rightarrow \operatorname{Der} \mathcal{A}_{0}$ is real linear. Let $f, g, h \in X$, $\kappa \in \mathbb{R} \backslash\{0\}$ and $c \in \mathbb{R}$. Then

$$
\begin{aligned}
& \sigma(f+c g, h) R(\kappa, h)^{2} \\
& =\delta_{f+c g}(R(\kappa, h))=\delta_{f}(R(\kappa, h))+c \delta_{g}(R(\kappa, h)) \\
& =(\sigma(f, h)+c \sigma(g, h)) R(\kappa, h)^{2} .
\end{aligned}
$$

Hence $\sigma(f+c g, h)=\sigma(f, h)+c \sigma(g, h)$ and, by the antisymmetry of $\sigma$ established in Lemma 2.5. one also has $\sigma(h, f+c g)=\sigma(h, f)+c \sigma(h, g)$. Thus $\sigma$ is real linear in both entries. Furthermore, if there is some $g \in X$ such that $\sigma(f, g)=0$ for all $f \in X$ one also has $\delta_{f}(R(\mu, g))=0, f \in X$. If $\delta$ acts ergodically on $\mathcal{A}$ it follows that $g=0$ since otherwise $R(\mu, g)$ is different from a multiple of the identity. So the form $\sigma$ is non-degenerate in this case. We summarize these results.

2.7 Proposition Let $(X, \mathcal{A})$ be a flat Lie $C^{*}$-system, i.e. $X$ is a real abelian Lie algebra and the pair $(X, \mathcal{A})$ satisfies the conditions (I), (II) and (III). Then there is an antisymmetric bilinear form $\sigma: X \times X \rightarrow \mathbb{R}$ such that for $\lambda, \mu \in \mathbb{R} \backslash\{0\}, f, g \in X$

(i) $[R(\lambda, f), R(\mu, g)]=i \sigma(f, g) R(\lambda, f) R(\mu, g)^{2} R(\lambda, f)$

(ii) $\delta_{f}(R(\mu, g))=\sigma(f, g) R(\mu, g)^{2}$.

If $\delta$ acts ergodically, then $\sigma$ is non-degenerate, i.e. $(X, \sigma)$ is a symplectic space.

These results show that the underlying $\mathrm{C}^{*}$-algebraic structure determines a central extension of the Lie algebra $X$ which is fixed by the form $\sigma$. In a faithful irreducible representation of $\mathcal{A}$ this extension of $X$ manifests itself in the commutation relations of the generators of the action $\delta$, given in Lemma 2.5. The preceding proposition expresses these relations in representation independent $\mathrm{C}^{*}$-algebraic terms. 


\section{Cohomology}

In this section we continue our analysis of flat Lie $\mathrm{C}^{*}$-systems and establish relations for pseudoresolvents which express additivity and homogeneity properties of the underlying action $\delta$. Since the pseudo-resolvents are not uniquely fixed by condition (II), it is clear that we may have to adjust them to establish such a result. In the proofs we make use of standard arguments from cohomology theory, the main problem being the control of domains of the generators in the chosen Hilbert space representation of $\mathcal{A}$. For this, we need the following technical lemma.

3.1 Lemma Let $n \in \mathbb{N}$, let $f_{1}, \ldots, f_{n} \in X$ and let $\lambda_{1}, \ldots \lambda_{n} \in \mathbb{R} \backslash\{0\}$. The linear manifold $\mathcal{D}_{f_{1}, \ldots, f_{n}} \doteq R\left(\lambda_{n}, f_{n}\right) \cdots R\left(\lambda_{1}, f_{1}\right) \mathcal{H}$ is dense in $\mathcal{H}$; it neither depends on the choice of $\lambda_{1}, \ldots, \lambda_{n}$ nor on the particular order of $f_{1}, \ldots, f_{n}$. Moreover, $\mathcal{A}_{0} \mathcal{D}_{f_{1}, \ldots, f_{n}} \subset \mathcal{D}_{f_{1}, \ldots, f_{n}}, \mathcal{D}_{f_{1}, \ldots, f_{n}} \subset \mathcal{D}_{f_{1}, \ldots, f_{n-1}}$ and $\mathcal{D}_{f_{1}, \ldots, f_{n}} \subset \bigcap_{k=1, \ldots, n} \mathcal{D}_{f_{k}}$ is a core for all operators $G_{f_{k}}, k=1, \ldots, n$.

Proof: The first part of the statement follows by another application of the fact that resolvents are bounded operators with dense range. For the proof that the parameters in $\mathbb{R} \backslash\{0\}$ can be arbitrarily chosen we use induction. The statement is clear for $n=1$ so, in view of the induction hypothesis, it suffices to show that $\lambda_{n+1}$ can be replaced by any other parameter $\mu_{n+1}$ without changing the respective domain. To verify this one makes use of the resolvent equation

$$
R\left(\mu_{n+1}, f_{n+1}\right)=R\left(\lambda_{n+1}, f_{n+1}\right)\left(1+i\left(\lambda_{n+1}-\mu_{n+1}\right) R\left(\lambda_{n+1}, f_{n+1}\right)\right) .
$$

Since $\left(1+i\left(\lambda_{n+1}-\mu_{n+1}\right) R\left(\mu_{n+1}, f_{n+1}\right)\right) \in \mathcal{A}_{0}$ by condition (III) one arrives, by repeated application of Lemma 2.1, at the inclusion

$$
R\left(\mu_{n+1}, f_{n+1}\right) R\left(\lambda_{n}, f_{n}\right) \cdots R\left(\lambda_{1}, f_{1}\right) \mathcal{H} \subset R\left(\lambda_{n+1}, f_{n+1}\right) R\left(\lambda_{n}, f_{n}\right) \cdots R\left(\lambda_{1}, f_{1}\right) \mathcal{H}
$$

Interchanging $\lambda_{n+1}, \mu_{n+1}$ one obtains the opposite inclusion, proving the independence of the domain on the choice of parameters. For the proof that the order of the chosen elements of $X$ does not matter either, it suffices to show that one can permute $f_{n+1}$ and $f_{n}$ without changing the domain. Now according to Lemma 2.6 and condition (III)

$$
\begin{aligned}
& R\left(\lambda_{n}, f_{n}\right) R\left(\lambda_{n+1}, f_{n+1}\right) \\
& =R\left(\lambda_{n+1}, f_{n+1}\right) R\left(\lambda_{n}, f_{n}\right)\left(1-i \sigma\left(f_{n+1}, f_{n}\right) R\left(\lambda_{n}, f_{n}\right) R\left(\lambda_{n+1}, f_{n+1}\right)\right) \\
& \doteq R\left(\lambda_{n+1}, f_{n+1}\right) R\left(\lambda_{n}, f_{n}\right) A_{0}
\end{aligned}
$$

where $A_{0} \in \mathcal{A}_{0}$. By another application of Lemma 2.1 it thus follows that

$$
R\left(\lambda_{n}, f_{n}\right) R\left(\lambda_{n+1}, f_{n+1}\right) \cdots R\left(\lambda_{1}, f_{1}\right) \mathcal{H} \subset R\left(\lambda_{n+1}, f_{n+1}\right) R\left(\lambda_{n}, f_{n}\right) \cdots R\left(\lambda_{1}, f_{1}\right) \mathcal{H}
$$

According to the preceding step one can interchange $\lambda_{n}, \lambda_{n+1}$ in this inclusion and interchanging also the role of $f_{n}, f_{n+1}$ one obtains the opposite inclusion, proving equality. The proof of the independence features of the domains $\mathcal{D}_{f_{1}, \ldots f_{n}}$ with regard to the elements entering into their definition is therewith complete. 
The stability of $\mathcal{D}_{f_{1}, \ldots f_{n}}$ under the action of $\mathcal{A}_{0}$ follows by still another application of Lemma2.1. Hence, in particular, $\mathcal{D}_{f_{1}, \ldots, f_{n}} \subset \mathcal{D}_{f_{1}, \ldots, f_{n-1}}$. Finally, since $\mathcal{D}_{f_{1}, \ldots f_{n}}=R\left(\lambda, f_{n}\right) \mathcal{D}_{f_{1}, \ldots f_{n-1}}$ for arbitrary $\lambda \in \mathbb{R} \backslash\{0\}$, it is clear that this domain is a core for $G_{f_{n}}$. But, as it is invariant under permutations of the elements $f_{1}, \ldots, f_{n}$, it is a core for all generators $G_{f_{k}}, k=1, \ldots, n$.

Making use of this lemma we can establish the existence of generators of the action $\delta$ which are additive on $X$.

3.2 Lemma There is a function $\gamma: X \rightarrow \mathbb{R}$ such that the "improved" generators

$$
\underline{G}_{f} \doteq G_{f}-\gamma(f) 1, \quad f \in X
$$

are additive, r.e.

$$
\left(\underline{G}_{f}+\underline{G}_{g}\right) \Phi=\underline{G}_{f+g} \Phi, \quad f, g \in X, \Phi \in \mathcal{D}_{f, g, f+g} .
$$

Moreover, these generators have the same domain and commutation properties as the given ones. The resulting resolvents

$$
\underline{R}(\lambda, f) \doteq\left(i \lambda \mathbf{1}+\underline{G}_{f}\right)^{-1}=\left((i \lambda-\gamma(f)) \mathbf{1}+G_{f}\right)^{-1}, \quad f \in X, \quad \lambda \in \mathbb{R} \backslash\{0\},
$$

satisfy condition (1.2), Lemma 2.6 and they are elements of $\mathcal{A}_{0}$.

Proof: Let $f, g \in X, \Phi \in \mathcal{D}_{f, g, f+g}$ and $A_{0} \in \mathcal{A}_{0}$. Then

$$
\left[\left(G_{f}+G_{g}-G_{f+g}\right), A_{0}\right] \Phi=-i\left(\delta_{f}\left(A_{0}\right)+\delta_{g}\left(A_{0}\right)-\delta_{f+g}\left(A_{0}\right)\right) \Phi=0
$$

where we used Lemma 3.1 and Lemma 2.2. Thus by the generalized Schur's lemmma there is a constant $\xi(f, g) \in \mathbb{R}$, symmetric in $f, g$, such that

$$
\left(G_{f}+G_{g}-G_{f+g}\right) \Phi=\xi(f, g) \Phi \quad \text { for all } \quad \Phi \in \mathcal{D}_{f, g, f+g}
$$

Next, let $f, g, h \in X$ and pick any non-zero vector

$$
\Phi \in \mathcal{D}_{f, g, h, f+g, g+h, f+g+h} \subset \mathcal{D}_{f, g, f+g} \cap \mathcal{D}_{f+g, h, f+g+h} \cap \mathcal{D}_{g, h, g+h} \cap \mathcal{D}_{f, g+h, f+g+h} .
$$

Because of the associativity of the addition of operators on a common domain the preceding result entails

$$
\begin{aligned}
& \left(G_{f}+G_{g}+G_{h}\right) \Phi \\
& =\left(G_{f+g}+G_{h}+\xi(f, g) 1\right) \Phi=G_{f+g+h} \Phi+(\xi(f, g)+\xi(f+g, h)) \Phi \\
& =\left(G_{f}+G_{g+h}+\xi(g, h) 1\right) \Phi=G_{f+g+h} \Phi+(\xi(f, g+h)+\xi(g, h)) \Phi .
\end{aligned}
$$

Hence $\xi: X \times X \rightarrow \mathbb{R}$ satisfies the cocycle equation

$$
\xi(f, g)+\xi(f+g, h)=\xi(f, g+h)+\xi(g, h), \quad f, g, h \in X
$$

It is well known that for any abelian group $X$, all real symmetric solutions $\xi$ of this equation are coboundaries [1]. More concretely, for any such $\xi$ there is a function $\gamma: X \rightarrow \mathbb{R}$ such that 
$\xi(f, g)=\gamma(f)+\gamma(g)-\gamma(f+g), f, g \in X$. This is the $\gamma$ in the statement of the lemma, because $\underline{G}_{f} \doteq G_{f}-\gamma(f) \mathbf{1}, f \in X$, has the same domain and commutation properties as $G_{f}$, and

$$
\left(\underline{G}_{f}+\underline{G}_{g}\right) \Phi=G_{f+g} \Phi+(\xi(f, g)-\gamma(f)-\gamma(g)) \Phi=\underline{G}_{f+g} \Phi
$$

for $f, g \in X$ and $\Phi \in \mathcal{D}_{f, g, f+g}$. This establishes the claim (3.1). Since $\underline{R}(\lambda, f)=R(\lambda+i \gamma(f), f)$ is contained in $\{R(z, f): z \in \mathbb{C} \backslash i \mathbb{R}, f \in X\}$, the last claim is also clear.

The next result expresses the additivity property (3.1) in terms of the modified resolvents.

3.3 Lemma Let $f, g \in X$ and let $\lambda, \mu,(\lambda+\mu) \in \mathbb{R} \backslash\{0\}$. Then

$$
\underline{R}(\lambda+\mu, f+g)\left(\underline{R}(\lambda, f)+\underline{R}(\mu, g)+i \sigma(f, g) \underline{R}(\lambda, f)^{2} \underline{R}(\mu, g)\right)=\underline{R}(\lambda, f) \underline{R}(\mu, g) \text {. }
$$

Proof: Let $\Phi \in \mathcal{D}_{f, g, f+g}$, then

$$
\begin{aligned}
& \underline{R}(\lambda+\mu, f+g)(\underline{R}(\lambda, f)+\underline{R}(\mu, g)) \Phi \\
& =\underline{R}(\lambda+\mu, f+g) \underline{R}(\lambda, f)\left(i(\lambda+\mu) 1+\underline{G}_{f}+\underline{G}_{g}\right) \underline{R}(\mu, g) \Phi \\
& =\left(\underline{R}(\lambda, f) \underline{R}(\mu, g)+\underline{R}(\lambda+\mu, f+g)\left[\underline{R}(\lambda, f), \underline{G}_{f+g}\right] \underline{R}(\mu, g)\right) \Phi \\
& =\left(\underline{R}(\lambda, f) \underline{R}(\mu, g)+\underline{R}(\lambda+\mu, f+g)\left(i \sigma(g, f) \underline{R}(\lambda, f)^{2}\right) \underline{R}(\mu, g)\right) \Phi,
\end{aligned}
$$

where in the second equality relation (3.1) was used and in the third one Lemmata 2.1 and 2.6. The statement then follows.

By condition (I), the map $\delta: X \rightarrow$ Der $\mathcal{A}_{0}$ is linear, and this raises the question whether there are underlying generators which are not only additive but also homogenous on $X$. An affirmative answer is given in the following lemma.

3.4 Lemma There is a function $\vartheta: X \rightarrow \mathbb{R}$ such that the "improved" generators

$$
\bar{G}_{f} \Phi \doteq\left(\underline{G}_{f}-\vartheta(f) 1\right) \Phi, \quad \Phi \in \mathcal{D}_{f} .
$$

are real linear ı.e.

$$
\left(\bar{G}_{f}+c \bar{G}_{g}\right) \Phi=\bar{G}_{f+c g} \Phi, \quad f, g \in X, c \in \mathbb{R}, \Phi \in \mathcal{D}_{f, g, f+c g}
$$

and they have the same domain and commutation properties as $G_{f}$. The resulting resolvents

$$
\bar{R}(\lambda, f) \doteq\left(i \lambda 1+\bar{G}_{f}\right)^{-1}=\left((i \lambda-\gamma(f)-\vartheta(f)) 1+G_{f}\right)^{-1}
$$

satisfy condition (1.2), Lemma 2.6 and Lemma 3.3 and they are elements of $\mathcal{A}_{0}$.

Proof: Every vector space has a Hamel basis. Thus for $X$ there is some index set $I$ and a subset $\left\{h_{\iota} \in X: \iota \in I\right\}$, such that every element $f \in X$ can be represented in a unique way as a finite sum $f=\sum_{\iota} c_{\iota}^{f} h_{\iota}$ with coefficients $c_{\iota}^{f} \in \mathbb{R}, \iota \in I$. This basis will be kept fixed below.

We prove homogeneity in analogy to relation (3.1). Let $f \in X, c \in \mathbb{R}, \Phi \in \mathcal{D}_{f, c f}$ and $A_{0} \in \mathcal{A}_{0}$, then by linearity of $\delta: X \rightarrow \operatorname{Der} \mathcal{A}_{0}$ we have

$$
\left[\left(c G_{f}-G_{c f}\right), A_{0}\right] \Phi=\left(c \delta_{f}\left(A_{0}\right)-\delta_{c f}\left(A_{0}\right)\right) \Phi=0 .
$$


Hence by the generalized Schur's lemma we get that for given $\iota \in I$ and $c \in \mathbb{R}$ there is some number $\zeta_{\iota}(c) \in \mathbb{R}$ such that

$$
\left(\underline{G}_{c h_{\iota}}-c \underline{G}_{h_{\iota}}\right) \Phi=\zeta_{\iota}(c) \Phi, \quad \Phi \in \mathcal{D}_{h_{\iota}, c h_{\iota}} .
$$

Clearly $\zeta_{\iota}(1)=0$, and $\zeta_{\iota}(0)=0$ since $\underline{G}_{0}=\zeta_{\iota}(0) \mathbf{1}$ on $\mathcal{D}_{h_{\iota}}$, and $\underline{G}_{f}$ is additive in $f$. Using this additivity, we also obtain for $c, c^{\prime} \in \mathbb{R}$ and $\Phi \in \mathcal{D}_{h_{\iota}, c h_{\iota}, c^{\prime} h_{\iota},\left(c+c^{\prime}\right) h_{\iota}}$ that

$$
\begin{aligned}
\zeta_{\iota}\left(c+c^{\prime}\right) \Phi & =\left(\underline{G}_{\left(c+c^{\prime}\right) h_{\iota}}-\left(c+c^{\prime}\right) \underline{G}_{h_{\iota}}\right) \Phi \\
& =\left(\underline{G}_{c h_{\iota}}-c \underline{G}_{h_{\iota}}+\underline{G}_{c^{\prime} h_{\iota}}-c^{\prime} \underline{G}_{h_{\iota}}\right) \Phi \\
& =\left(\zeta_{\iota}(c)+\zeta_{\iota}\left(c^{\prime}\right)\right) \Phi .
\end{aligned}
$$

Hence $\zeta_{\iota}: \mathbb{R} \rightarrow \mathbb{R}$ is additive. Now let $f \in X$ with corresponding decomposition $f=\sum_{\iota} c_{\iota}^{f} h_{\iota}$. Since this decomposition is unique and only a finite number of coefficients $c_{\iota}^{f}$ are nonzero we may define $\vartheta(f) \doteq \sum_{\iota} \zeta_{\iota}\left(c_{\iota}^{f}\right)$ which produces a map $\vartheta: X \rightarrow \mathbb{R}$. Let $g=\sum_{\iota} c_{\iota}^{g} h_{\iota} \in X$, then $f+g=\sum_{\iota}\left(c_{\iota}^{f}+c_{\iota}^{g}\right) h_{\iota}$ and so

$$
\vartheta(f+g)=\sum_{\iota} \zeta_{\iota}\left(c_{\iota}^{f}+c_{\iota}^{g}\right)=\sum_{\iota}\left(\zeta_{\iota}\left(c_{\iota}^{f}\right)+\zeta_{\iota}\left(c_{\iota}^{g}\right)\right)=\vartheta(f)+\vartheta(g)
$$

hence $\vartheta$ is additive. Since $\underline{G}_{f}$ is also additive in $f$, it follows that the operators $\bar{G}_{f}: \mathcal{D}_{f} \rightarrow \mathcal{H}$ given by

$$
\bar{G}_{f} \Phi \doteq\left(\underline{G}_{f}-\vartheta(f) \mathbf{1}\right) \Phi, \quad \Phi \in \mathcal{D}_{f} .
$$

are additive as well. They have the same domain and commutation properties as $G_{f}$.

For the proof that the generators $\bar{G}(c f), c \in \mathbb{R}$, are homogenous in $c$, consider first the case where $f=h_{\iota}$, in which case $\vartheta\left(c h_{\iota}\right)=\zeta_{\iota}(c)$ and $\vartheta\left(h_{\iota}\right)=\zeta_{\iota}(1)=0$. Then

$$
\left(\bar{G}_{c h_{\iota}}-c \bar{G}_{h_{\iota}}\right) \Phi=\left(\left(\underline{G}_{c h_{\iota}}-\zeta_{\iota}(c) \mathbf{1}\right)-c\left(\underline{G}_{h_{\iota}}-\zeta_{\iota}(1) \mathbf{1}\right)\right) \Phi=0
$$

making use of (3.3) and $\zeta_{\iota}(1)=0$. For the general case we make use of the full power of Lemma 3.1 for arbitrary $n \in \mathbb{N}$. Let $f=\sum_{\iota} c_{\iota}^{f} h_{\iota}$. Since only a finite number of the terms $c_{\iota}^{f} h_{\iota}$ is different from zero there is some dense domain $\mathcal{D} \subset \mathcal{D}_{f, c f}$ which is stable under the action of $\mathcal{A}_{0}$ and it lies in the domains of all generators $G_{k}$ with $k \in\left\{c_{\iota}^{f} h_{\iota}, c c_{\iota}^{f} h_{\iota}: \iota \in I\right\}$ as well as $k$ being a sum of these. Hence one obtains for $\Phi \in \mathcal{D}$,

$$
\begin{aligned}
\left(\bar{G}_{c f}-c \bar{G}_{f}\right) \Phi & =\left(\bar{G}_{\sum_{\iota} c c_{\iota}^{f} h_{\iota}}-c \bar{G}_{\sum_{\iota} c_{\iota}^{f} h_{\iota}}\right) \Phi \\
& =\sum_{\iota}\left(\bar{G}_{c c_{\iota}^{f} h_{\iota}}-c \bar{G}_{c_{\iota}^{f} h_{\iota}}\right) \Phi=\sum_{\iota}\left(c c_{\iota}^{f} \bar{G}_{h_{\iota}}-c c_{\iota}^{f} \bar{G}_{h_{\iota}}\right) \Phi=0,
\end{aligned}
$$

where in the second equality the additivity of $\bar{G}$ was used and in the third equality homogeneity w.r.t. $h_{\iota}$. Since $\mathcal{D}$ is a core for the underlying generators, it follows that $\bar{G}_{c f}=c \bar{G}_{f}$ on $\mathcal{D}_{f, c f}$.

As before we define the improved resolvents

$$
\bar{R}(\lambda, f) \doteq\left(i \lambda \mathbf{1}+\bar{G}_{f}\right)^{-1}=\left((i \lambda-\gamma(f)-\vartheta(f)) \mathbf{1}+G_{f}\right)^{-1}
$$

which still satify condition (1.2) and Lemma 2.6. Since the generators $\bar{G}$ are additive, the corresponding resolvents also satisfy the relation given in Lemma 3.3. The last claim is clear in view of condition (III). 
The homogeneity of the generators manifests itself in further algebraic properties.

3.5 Lemma Let $f \in X$ and let $\lambda, c \in \mathbb{R} \backslash\{0\}$. Then

$$
c \bar{R}(c \lambda, c f)=\bar{R}(\lambda, f)
$$

Proof: Pick any vector $\Phi \in \mathcal{D}_{f, c f}$, then it follows from Lemma 3.1 that

$$
\begin{aligned}
& (c \bar{R}(c \lambda, c f)-\bar{R}(\lambda, f)) \Phi \\
& =\bar{R}(c \lambda, c f)(c(i \lambda \mathbf{1}+\bar{G}(f))-(i c \lambda \mathbf{1}+\bar{G}(c f))) \bar{R}(\lambda, f) \Phi=0,
\end{aligned}
$$

where the second equality follows from the homogeneity of the generators.

We summarize our findings. Let $\mathcal{R} \subset \mathcal{A}$ be the $\mathrm{C}^{*}$-algebra generated by the range of the underlying resolvents $R$. In the preceding discussion we have shown that one can proceed from these resolvents by analytic continuation to improved resolvents $\bar{R} \in \mathcal{R}$, in which the vector space structure of $X$ manifests itself by additional relations. We have worked in a concrete representation of $\mathcal{A}$. But since this representation was faithful the above results can be reformulated in the abstract setting.

3.6 Theorem Let $(X, \mathcal{A})$ be a flat Lie $C^{*}$-system, i.e. $X$ is a real abelian Lie algebra and the pair $(X, \mathcal{A})$ satisfies the conditions (I), (II) and (III). Let $\mathcal{R} \subset \mathcal{A}$ be the $C^{*}$-algebra generated by the corresponding pseudo-resolvents. There are a skew-symmetric bilinear form $\sigma: X \times X \rightarrow \mathbb{R}$ and pseudo-resolvents $\{\bar{R}(\lambda, f): \lambda \in \mathbb{R} \backslash\{0\}, f \in X\} \subset \mathcal{R} \cap \mathcal{A}_{0}$ such that

$$
\bar{R}(\lambda, f) \delta_{f}\left(A_{0}\right) \bar{R}(\lambda, f)=i\left[A_{0}, \bar{R}(\lambda, f)\right], \quad A_{0} \in \mathcal{A}_{0}
$$

and, for $f, g \in X, \lambda, \mu \in \mathbb{R} \backslash\{0\}$ and $\lambda+\mu \neq 0$ in item (ii),

(i) $[\bar{R}(\lambda, f), \bar{R}(\mu, g)]=i \sigma(f, g) \bar{R}(\lambda, f) \bar{R}(\mu, g)^{2} \bar{R}(\lambda, f)$

(ii) $\bar{R}(\lambda+\mu, f+g)\left(\bar{R}(\lambda, f)+\bar{R}(\mu, g)+i \sigma(f, g) \bar{R}(\lambda, f)^{2} \bar{R}(\mu, g)\right)=\bar{R}(\lambda, f) \bar{R}(\mu, g)$

(iii) $c \bar{R}(c \lambda, c f)=\bar{R}(\lambda, f)$ for $c \in \mathbb{R} \backslash\{0\}$

(iv) $\delta_{f}(\bar{R}(\mu, g))=\sigma(f, g) \bar{R}(\mu, g)^{2}, \quad \mu \in \mathbb{R} \backslash\{0\}$.

If $\delta$ acts ergodically on $\mathcal{A}$, then $\sigma$ is non-degenerate.

As the range of the analytic continuations of the resolvents is contained in $\mathcal{R}$, this algebra is generated by the improved resolvents as well. Moreover, if $\delta$ acts ergodically, the relations obtained above show that the algebra $\mathcal{R}$ is just the resolvent algebra $\mathcal{R}(X, \sigma)$, defined in [2]. It is noteworthy that $\mathcal{R}(X, \sigma)$ is primitive since its Fock representation is faithful. Finally, we show that in general the algebra $\mathcal{R}$ is independent from the choice of pseudo-resolvents satisfying relation (1.2). 
3.7 Proposition Let $(X, \mathcal{A})$ be a flat Lie $C^{*}$-system and let $\left\{R(\lambda, f) \in \mathcal{A}_{0}: \lambda \in \mathbb{R} \backslash\{0\}, f \in X\right\}$ and $\left\{R^{\prime}(\lambda, f) \in \mathcal{A}_{0}: \lambda \in \mathbb{R} \backslash\{0\}, f \in X\right\}$ be two families of pseudo-resolvents satisfying relation (1.2). Then the respective $C^{*}$-algebras generated by these families coincide.

Proof: As before, assume without loss of generality that we have concretely $\mathcal{A} \subseteq \mathcal{B}(\mathcal{H})$ for some Hilbert space $\mathcal{H}$ and $\mathcal{A}^{-}=\mathcal{B}(\mathcal{H})$, where the bar denotes weak closure. Thus there are selfadjoint generators $G_{f}$ (resp. $G_{f}^{\prime}$ ) which are densely defined on the domain $\mathcal{D}_{f} \doteq R(\lambda, f) \mathcal{H}$ (resp. $\left.\mathcal{D}_{f}^{\prime} \doteq R^{\prime}(\lambda, f) \mathcal{H}\right)$ and satisfy $R(\lambda, f)=\left(i \lambda \mathbf{1}+G_{f}\right)^{-1}\left(\operatorname{resp} . R^{\prime}(\lambda, f)=\left(i \lambda \mathbf{1}+G_{f}^{\prime}\right)^{-1}\right)$.

Let $\mathcal{D} \doteq R(\lambda, f) R^{\prime}(\lambda, f) \mathcal{H}$ for fixed $\lambda \in \mathbb{R} \backslash\{0\}, f \in X$. Since resolvents are bounded and their range is dense, it is clear that $\mathcal{D}$ is dense in $\mathcal{H}$. By Lemma 2.1, we also have that for any $A_{0} \in \mathcal{A}_{0}$ there is a $B_{0} \in \mathcal{A}_{0}$ such that $A_{0} R(\lambda, f)=R(\lambda, f) B_{0}$ and also the analogous statement for $R^{\prime}(\lambda, f)$. Thus we obtain stability of $\mathcal{D}$ under the action of $\mathcal{A}_{0}$. By definition $\mathcal{D} \subset \mathcal{D}_{f}$ is a core for $G_{f}$. If we let $A_{0}=R(\lambda, f)$ then $R(\lambda, f) R^{\prime}(\lambda, f)=R^{\prime}(\lambda, f) B_{0}$ for some $B_{0} \in \mathcal{A}_{0}$, hence $\mathcal{D} \subset R^{\prime}(\lambda, f) \mathcal{H}=\mathcal{D}_{f}^{\prime}$. Moreover, as $R^{\prime}(\lambda, f) \in \mathcal{A}_{0}$ it is also clear that $R^{\prime}(\lambda, f) \mathcal{D} \subset \mathcal{D}$, and hence $\mathcal{D}$ is a core for $G_{f}^{\prime}$ as well. Now observe that for $\Phi \in \mathcal{D}$ and $A_{0} \in \mathcal{A}_{0}$ we have via Lemma 2.2 that

$$
\left[\left(G_{f}^{\prime}-G_{f}\right), A_{0}\right] \Phi=-i\left(\delta_{f}\left(A_{0}\right)-\delta_{f}\left(A_{0}\right)\right) \Phi=0 .
$$

Thus by the generalized Schur's lemma there is some $c_{f} \in \mathbb{R}$ such that $\left(G_{f}^{\prime}-G_{f}-c_{f} \mathbf{1}\right) \uparrow \mathcal{D}=0$. Since $\mathcal{D}$ is a core for $G_{f}$ and $G_{f}^{\prime}$ it follows that $G_{f}^{\prime}=G_{f}+c_{f} \mathbf{1}$, proving that

$$
R^{\prime}(\lambda, f)=\left(\left(i \lambda+c_{f}\right) \mathbf{1}+G_{f}\right)^{-1} \in\{R(z, f): z \in \mathbb{C} \backslash i \mathbb{R}\} \subset \mathcal{R} .
$$

Thus the $\mathrm{C}^{*}$-algebra generated by $\left\{R^{\prime}(\lambda, f) \in \mathcal{A}_{0}: \lambda \in \mathbb{R} \backslash\{0\}, f \in X\right\}$ is contained in the $\mathrm{C}^{*}$-algebra generated by $\left\{R(\lambda, f) \in \mathcal{A}_{0}: \lambda \in \mathbb{R} \backslash\{0\}, f \in X\right\}$ and by symmetry of the argument we also have the reverse inclusion, hence equality.

\section{Concluding remarks}

We have established above a $\mathrm{C}^{*}$-algebraic framework for the systematic study of the representation theory of Lie-algebras of derivations tailored to the needs of quantum physics. For the simple case of flat Lie $\mathrm{C}^{*}$-systems $(X, \mathcal{A})$ arising from actions of abelian Lie algebras $X$ on primitive $\mathrm{C}^{*}$-algebras $\mathcal{A}$, we were able to completely determine the algebraic structure of the generators. It turned out that this structure provides in general a central extension of $X$ whose specific form is fixed by some skew-symmetric bilinear form $\sigma: X \times X \rightarrow \mathbb{R}$ encoded in the underlying algebraic data. Remarkably, the $\mathrm{C}^{*}$-algebra generated by the resolvents of the generators coincides with the resolvent algebra $\mathcal{R}(X, \sigma)$, invented in [2] as a convenient framework for the description of quantum systems.

In view of these results it seems worthwhile to extend this study of representations of Lie algebras of derivations to the non-abelian case. It has to be noted that the technical condition (III) would no longer be meaningful in this general context, i.e. the pseudo-resolvents do not need to belong to the domain of the action $\delta$. As a matter of fact, as these pseudo-resolvents are 
assumed to be elements of $\mathcal{A}$, cf. condition (II), one may even have to relax the assumption that the domain $\mathcal{A}_{0}$ of $\delta$ is norm dense in $\mathcal{A}$. It would still be meaningful to require that this domain is weakly dense in all faithful representations of $\mathcal{A}$. In fact, the present results can be established under this weaker assumption. A solution of these mathematical problems would be rewarding since it would shed new light on the appearance of central extensions of symmetry groups in the context of quantum physics.

\section{Acknowledgements}

DB and HG are grateful for the support of the Courant Research Center "Higher Order Structures" of the University of Göttingen

\section{References}

[1] J. Aczél, The general solution of two functional equations by reduction to functions additive in two variables and with the aid of a Hamel basis, Glasnik Mat.-Fiz. Astronom., 20, 65-73 (1965).

[2] D. Buchholz and H. Grundling, The Resolvent Algebra: A New Approach to Canonical Quantum Systems. J. Funct. Analysis 254, 2725-2779 (2008)

[3] D. Buchholz and H. Grundling, Algebraic Supersymmetry: A Case study. Commun. Math. Phys. 272, 699-750 (2007)

[4] Dixmier, J.: C*-algebras, North Holland Publishing Company, Amsterdam - New York Oxford 1977

[5] Kato, T.: Remarks on Pseudo-resolvents and Infinitesimal Generators of Semi-groups. Proc. Japan Acad. 35 (1959), 467-468.

[6] S. Sakai, $C^{*}$-algebras and $W^{*}$-algebras, Springer (1971)

[7] K. Yosida, Functional Analysis, Springer (1980) 